PROCEEDINGS OF THE

AMERICAN MATHEMATICAL SOCIETY

Volume 134, Number 10, October 2006, Pages 2849-2856

S 0002-9939(06)08335-3

Article electronically published on May 2, 2006

\title{
QUASI-TRANSLATIONS AND COUNTEREXAMPLES TO THE HOMOGENEOUS DEPENDENCE PROBLEM
}

\author{
MICHIEL DE BONDT
}

(Communicated by Bernd Ulrich)

\begin{abstract}
In this article, the author gives counterexamples to the Linear Dependence Problem for Homogeneous Nilpotent Jacobians for dimension 5 and up. This problem has been formulated as a conjecture/problem by several authors in connection to the Jacobian conjecture. In dimension 10 and up, cubic counterexamples are given.

In the construction of these counterexamples, the author makes use of socalled quasi-translations, a special type of invertible polynomial maps. Quasitranslations can also be seen as a special type of locally nilpotent derivations.
\end{abstract}

\section{INTRODUCTION}

Write $\mathcal{J} H$ for the Jacobian of $H$. The Linear Dependence Problem for Homogeneous Nilpotent Jacobians asserts that, if $H=\left(H_{1}, H_{2}, \ldots, H_{n}\right)$ is a homogeneous polynomial map such that $\mathcal{J} H$ is nilpotent, then the rows of $\mathcal{J} H$ are dependent over $\mathbb{C}$, i.e. there are $\lambda_{i} \in \mathbb{C}$, not all zero, such that

$$
\lambda_{1} \mathcal{J} H_{1}+\lambda_{2} \mathcal{J} H_{2}+\ldots+\lambda_{n} \mathcal{J} H_{n}=0
$$

or equivalently, the components of $H$ are linearly dependent over $\mathbb{C}$, i.e. there are $\lambda_{i} \in \mathbb{C}$, not all zero, such that

$$
\lambda_{1} H_{1}+\lambda_{2} H_{2}+\ldots+\lambda_{n} H_{n}=0 .
$$

Let $d$ be the degree of $H$. Positive answers to the Linear Dependence Problem for Homogeneous Nilpotent Jacobians are known in the following cases:

- $\operatorname{rk} \mathcal{J} H \leq 1$ (including the case $n \leq 2$, also if $H$ is not homogeneous; see [1] or 7]),

- $n=3, d$ arbitrary (by van den Essen and the author; see [4]),

- $n=4, d \leq 3$ (by Hubbers; see [10] or [7]).

I will give counterexamples to the Linear Dependence Problem for Homogeneous Nilpotent Jacobians in dimension 5 and up, including cubic counterexamples in dimension 10 and up. On the Cubic Linear Dependence Problem for Homogeneous Nilpotent Jacobians, C. Olech put up a bottle of Polish vodka for the one who first solved the problem by way of either a proof or a counterexample; see [12.

Received by the editors October 5, 2004 and, in revised form, April 27, 2005.

2000 Mathematics Subject Classification. Primary 14R15, 14R20, 14R99.

Key words and phrases. Jacobian (conjecture), quasi-translation, linear dependence problem. The author was supported by the Netherlands Organization of Scientific Research (NWO).

(C)2006 American Mathematical Society Reverts to public domain 28 years from publication 
Write $x=\left(x_{1}, x_{2}, \ldots, x_{n}\right)$. We call the polynomial map $x+H$ a quasi-translation if its polynomial inverse is $x-H$. In section 11 I will show that for quasi-translations $x+H, \mathcal{J} H$ is nilpotent. I will construct counterexamples of the quasi-translation type to the Linear Dependence Problem for Homogeneous Nilpotent Jacobians in dimension 6 and up. For these counterexamples $H=\left(H_{1}, H_{2}, \ldots, H_{n}\right)$, the associated derivation

$$
D=\sum_{i=1}^{n} H_{i} \frac{\partial}{\partial x_{i}}
$$

is locally nilpotent, such that $\operatorname{ker} D$ does not contain coordinates. In particular, it gives a new class of locally nilpotent derivations of maximal rank. The first examples of such derivations were constructed by Freudenburg in [8].

\section{Quasi-Translations}

Let $H=\left(H_{1}, H_{2}, \ldots, H_{n}\right)$ be a polynomial map and let $D=\sum_{i=1}^{n} H_{i} \frac{\partial}{\partial x_{i}}$ be the derivation associated with it. $H$ does not need to be homogeneous yet.

The following proposition makes it clear that quasi-translations can be seen as a special kind of both nilpotent Jacobians and locally nilpotent derivations.

Proposition 1.1. $x+H$ is a quasi-translation, if and only if $D^{2} x_{i}=0$ for all $i$. Furthermore, $\mathcal{J} H$ is nilpotent in the case $x+H$ is a quasi-translation.

Proof. i) Assume $x+H$ is a quasi-translation. Then $(x-H) \circ(x+H)=x$, whence

$$
H(x+H)=(x-(x-H)) \circ(x+H)=x+H-x=H
$$

and

$$
H(x+(m+1) H)=H(x+H+m H)=H(x+H+m H(x+H)) .
$$

Assume that $H(x+m H)=H(x)$. Substituting $x=x+H$ in it gives

$$
H(x+(m+1) H)=H(x+H+m H(x+H))=H(x+H)=H,
$$

whence

$$
H(x+m H)=H
$$

follows for all $m \in \mathbb{N}$ by induction. Consequently,

$$
H(x+t H)=H
$$

is an equality of polynomial maps, where $t$ is a new indeterminate. If we first differentiate (11) to $t$ and then substitute $t=0$, then we get

$$
\mathcal{J} H \cdot H=0
$$

which is equivalent to $D^{2} x_{i}=D H_{i}=0$ for all $i$.

ii) Assume $D^{2} x_{i}=0$ for all $i$. Then $H_{i} \in \operatorname{ker} D$ for all $i$, whence

$$
H_{i}=(\exp D) H_{i}=H_{i}\left((\exp D) x_{1}, \ldots,(\exp D) x_{n}\right)=H_{i}(x+H)
$$

and

$$
(x-H) \circ(x+H)=x+H-H(x+H)=x+H-H=x .
$$

iii) Since $x-t H$ is an invertible polynomial map, the entries of

$$
(\mathcal{J}(x-t H))^{-1}=\left(I_{n}-t \mathcal{J} H\right)^{-1}=I_{n}+t \mathcal{J} H+t^{2}(\mathcal{J} H)^{2}+\cdots
$$

are contained in $\mathbb{C}[t]$. It follows that $\mathcal{J} H$ is nilpotent.

This completes the proof of Proposition 1.1. 
The following proposition characterizes the homogeneous relations between the components of $H$.

Proposition 1.2. Let $g$ be homogeneous of degree e. Then $D^{e+1} g=0$. Furthermore, $D^{e} g=0$, if and only if $g(H)=0$.

Proof. Looking at the coefficient of $t^{e}$ in

$$
(\exp t D) g=g\left((\exp t D) x_{1}, \ldots,(\exp t D) x_{n}\right)=g\left(x_{1}+t H_{1}, \ldots, x_{n}+t H_{n}\right)
$$

we get

$$
D^{e} g=e ! g(H),
$$

and the assertions of Proposition 1.2 follow.

Corollary 1.3. A linear form $R$ is a linear relation between the components of $H$, if and only if $R \in \operatorname{ker} D$.

The above corollary shows that quasi-translations $x+H$ without linear relations between the components of $H$ are a special type of locally nilpotent derivations without linear kernel elements. We will construct such quasi-translations.

\section{HOMOGENEOUS QUASI-TRANSLATIONS WITH LINEARLY INDEPENDENT COMPONENTS}

Put

$$
H_{A, B}^{[n]}:=\left(\begin{array}{c}
B\left(A x_{1}-B x_{2}\right) \\
A\left(A x_{1}-B x_{2}\right) \\
B\left(A x_{3}-B x_{4}\right) \\
A\left(A x_{3}-B x_{4}\right) \\
\vdots \\
B\left(A x_{n-1}-B x_{n}\right) \\
A\left(A x_{n-1}-B x_{n}\right)
\end{array}\right) \quad(n \text { even })
$$

where $A$ and $B$ are indeterminates so that

$$
D_{A, B}^{[n]}:=\sum_{i=1}^{n}\left(H_{A, B}^{[n]}\right)_{i} \frac{\partial}{\partial x_{i}}
$$

is a derivation on the ring $\mathbb{C}[A, B, x]$. Then $\left(\mathcal{J} H_{A, B}^{[n]}\right)^{2}=0$, for

$$
\mathcal{J} H_{A, B}^{[n]}=\left(\begin{array}{ccccc}
A B & -B^{2} & & & \emptyset \\
A^{2} & -A B & & & \\
& & \ddots & & \\
& & & A B & -B^{2} \\
\emptyset & & & A^{2} & -A B
\end{array}\right) .
$$

Since $H_{A, B}^{[n]}$ is homogeneous of degree 1, it follows from Euler's formula that

$$
\mathcal{J} H_{A, B}^{[n]} \cdot H_{A, B}^{[n]}=\mathcal{J} H_{A, B}^{[n]} \cdot \mathcal{J} H_{A, B}^{[n]} \cdot x=0 .
$$

Therefore, $D_{A, B}^{[n]}$ satisfies

$$
\left(D_{A, B}^{[n]}\right)^{2} x_{i}=0
$$

for all $i$. 
If $i, j \leq n / 2$, then

$$
\begin{aligned}
D_{A, B}^{[n]}\left(x_{2 i-1} x_{2 j}\right) & =\left(H_{A, B}^{[n]}\right)_{2 i-1} x_{2 j}+x_{2 i-1}\left(H_{A, B}^{[n]}\right)_{2 j} \\
& =B\left(A x_{2 i-1}-B x_{2 i}\right) x_{2 j}+x_{2 i-1} A\left(A x_{2 j-1}-B x_{2 j}\right) \\
& =-B^{2} x_{2 i} x_{2 j}+A^{2} x_{2 i-1} x_{2 j-1} .
\end{aligned}
$$

The right-hand side is symmetric in $i, j$, whence

$$
x_{2 i-1} x_{2 j}-x_{2 i} x_{2 j-1} \in \operatorname{ker} D_{A, B}^{[n]} \quad(i, j \leq n / 2) .
$$

Now assume that $n \geq 6$ even and take

$$
a:=x_{1} x_{4}-x_{2} x_{3}
$$

and

$$
b:=x_{3} x_{6}-x_{4} x_{5} .
$$

Since $a, A, b, B \in \operatorname{ker} D_{A, B}^{[n]}, D_{A, B}^{[n]}$ induces a derivation $D_{a, b}^{[n]}$ on $\mathbb{C}[A, B, x] /$ $(A-a, B-b)=\mathbb{C}[x]$, for which $a, b \in \operatorname{ker} D_{a, b}^{[n]}$. From (44), it follows that

$$
\left(D_{a, b}^{[n]}\right)^{2} x_{i}=0
$$

for all $i$.

Put

$$
c:=x_{1} x_{6}-x_{2} x_{5}
$$

and

$$
f:=c\left(a x_{n-1}-b x_{n}\right) .
$$

Since $D_{a, b}^{[n]}$ is locally nilpotent, $\operatorname{ker} D_{a, b}^{[n]}$ is factorially closed. From $c \in \operatorname{ker} D$, $\left(H_{a, b}^{[n]}\right)_{n} \in \operatorname{ker} D_{a, b}^{[n]}$ and $\left(a x_{n-1}-b x_{n}\right) \mid\left(H_{a, b}^{[n]}\right)_{n}, f \in \operatorname{ker} D_{a, b}^{[n]}$, follows.

Theorem 2.1. Assume $n \geq 6$ even. Then $x+H_{a, b}^{[n]}$ and $\left(x, x_{n+1}\right)+\left(H_{a, b}^{[n]}, f\right)$ are quasi-translations, for which there are no linear relations between the components of $H_{a, b}^{[n]}$ resp. $\left(H_{a, b}^{[n]}, f\right)$. Furthermore, $H_{a, b}^{[n]}$ resp. $\left(H_{a, b}^{[n]}, f\right)$ is homogeneous of degree 5.

Proof. We already saw that $x+H_{a, b}^{[n]}$ is a quasi-translation. Since $f \in \operatorname{ker} D_{a, b}^{[n]}$ and the components of $\left(H_{a, b}^{[n]}, f\right)$ are contained in $\mathbb{C}[x]\left(\right.$ no $\left.x_{n+1}\right), \mathcal{J}_{n+1}\left(H_{a, b}^{[n]}, f\right)^{2}$. $\left(x, x_{n+1}\right)=0$, i.e. $\left(x, x_{n+1}\right)+\left(H_{a, b}^{[n]}, f\right)$ is a quasi-translation.

So it remains to show the linear independence of the components of $H_{a, b}^{[n]}$ resp. $\left(H_{a, b}^{[n]}, f\right)$. For that purpose, define $y=\left(t, t^{2}-1, t^{3}, t^{4}, t^{5}-1, t^{6}, t^{7}, \ldots, t^{n}\right)$. Then $a(y)=t^{3}, b(y)=t^{4}$ and $c(y)=t^{5}+t^{2}-1$. It follows that for all $i \leq n / 2$,

$$
\begin{gathered}
\left(H_{a, b}^{[n]}\right)_{2 i-1}(y)=-t^{8+2 i}+O\left(t^{7+2 i}\right), \\
\left(H_{a, b}^{[n]}\right)_{2 i}(y)=-t^{7+2 i}+O\left(t^{6+2 i}\right)
\end{gathered}
$$

and

$$
f=-t^{9+n}+O\left(t^{8+n}\right) .
$$

Therefore, all components of $H_{a, b}^{[n]}(y)$ resp. $\left(H_{a, b}^{[n]}, f\right)(y)$ have different order in $t$, and the linear independence of the components of $H_{a, b}^{[n]}$ resp. $\left(H_{a, b}^{[n]}, f\right)$ follows.

Corollary 2.2. The Linear Dependence Problem for Homogeneous Jacobians has a negative answer in dimension 6 and up. 
In 1876 P. Gordan and M. Nöther had already proved that in dimension $n \leq 4$, no counterexamples of the quasi-translation type to the Linear Dependence Problem for Homogeneous Jacobians exist; see [9]. More generally, they showed that for a quasi-translation $x+H$ in any dimension $n \geq 3$, with $H$ homogeneous and $\operatorname{rk} \mathcal{J} H \leq$ 2 , there are even two independent linear combinations between the components of $H$. In proposition 1.2 of 4 , it is shown that $\operatorname{rk} \mathcal{J} H \leq n-2$ if $x+H$ is a quasitranslation with $H$ homogeneous and $n \geq 3$, whence $\operatorname{rk} \mathcal{J} H \leq 2$ in case $n \leq 4$.

P. Gordan and M. Nöther used geometric methods to get this result. In [5], the author gives an algebraic proof of a slightly more general result. P. Gordan and M. Nöther did some research in dimension 5 as well, the only dimension for which it is not yet known whether the components of $H$ need to be linearly dependent. In [5], it is shown that one only needs to consider quasi-translations $x+H$, where $H$ is of the form

$$
H=\left(h_{1}(p, q), h_{2}(p, q), h_{3}(p, q), h_{4}(p, q), r\right)
$$

with $h=\left(h_{1}, h_{2}, h_{3}, h_{4}\right)$ homogeneous and $p, q$ homogeneous of the same degree. In the spirit of C. Olech, I promise a bottle of Joustra Beerenburg (Frisian spirit) for the one who first solves the problem whether for quasi-translations $x+H$ in dimension 5 with $H$ homogeneous, the components of $H$ need to be linearly dependent.

Although in dimension 4 no counterexamples of the quasi-translation type exist to the Linear Dependence Problem for Homogeneous Nilpotent Jacobians, this problem is still open in dimension 4 for degree 4 and up.

\section{Other HOMOgEnEOUS JACOBIANS WITHOUT LINEAR DEPENDENCES}

The main theorem of this section is the following.

Theorem 3.1. Let $H=\left(H_{1}, H_{2}, \ldots, H_{s}, \ldots, H_{n}\right)$ and

$$
D=\sum_{i=1}^{s} H_{i} \frac{\partial}{\partial x_{i}}
$$

Assume that $H_{i} \in \operatorname{ker} D$ for all $i$ and $H_{i} \in \mathbb{C}\left[x_{1}, x_{2}, \ldots, x_{i-1}\right]$ for all $i>s$. Then $x+H$ is an invertible polynomial map. In particular, $\mathcal{J} H$ is nilpotent if $H$ is homogeneous of degree 2 at least.

Proof. Since $D^{2} x_{i}=0$ for all $i, H_{s+1} \in \operatorname{ker} D$ and $H_{s+1} \in \mathbb{C}\left[x_{1}, x_{2}, \ldots, x_{s}\right]$, we have

$$
\begin{aligned}
H_{s+1} & =(\exp D) H_{s+1} \\
& =H_{s+1}\left((\exp D) x_{1},(\exp D) x_{2}, \ldots,(\exp D) x_{s}\right) \\
& =H_{s+1}\left(x_{1}+H_{1}, x_{2}+H_{2}, \ldots, x_{s}+H_{s}\right) .
\end{aligned}
$$

Therefore, if we define the polynomial map $G_{i}$ as

$$
G_{i}=\left(x_{1}, x_{2}, \ldots, x_{i-1}, x_{i}-H_{i}, x_{i+1}, \ldots, x_{n}\right)
$$

for all $i>s$, then

$G_{s+1} \circ(x+H)=\left(x_{1}+H_{1}, x_{2}+H_{2}, \ldots, x_{s}+H_{s}, x_{s+1}, x_{s+2}+H_{s+2}, \ldots, x_{n}+H_{n}\right)$.

By induction on $s$,

$G_{n} \circ G_{n-1} \circ \cdots \circ G_{s+1} \circ(x+H)=\left(x_{1}+H_{1}, x_{2}+H_{2}, \ldots, x_{s}+H_{s}, x_{s+1}, x_{s+2}, \ldots, x_{n}\right)$ 
follows, which is the quasi-translation corresponding to $D$ and hence invertible. Consequently, $x+H$ is invertible, since each $G_{i}$ with $i>s$ is invertible. So $\operatorname{det}\left(I_{n}+\mathcal{J} H\right)=\operatorname{det} \mathcal{J}(x+H) \in \mathbb{C}^{*}$. This implies that $\mathcal{J} H$ is nilpotent in case $H$ is homogeneous of degree at least 2 (see [7, 6.2.11]).

Again, take $a:=x_{1} x_{4}-x_{2} x_{3}$ and $b:=x_{3} x_{6}-x_{4} x_{5}$.

Corollary 3.2. Put

$$
H=\left(x_{5} H_{a, x_{5}^{2}}^{[4]}, a^{3}\right) .
$$

Then $H$ is a counterexample in dimension 5 to the Linear Dependence Problem for Homogeneous Jacobians. Observe that $H$ has degree 6.

Proof. We apply Theorem 3.1 with $s=4$, so let

$$
D=\sum_{i=1}^{4} H_{i} \frac{\partial}{\partial x_{i}} .
$$

Then $D$ and $D_{a, x_{5}^{2}}^{[4]}$ have the same kernel, which contains $x_{5}$. It follows that $D^{2} x_{i}=0$ for all $i$. Furthermore, $a^{3} \in \operatorname{ker} D$ and $a^{3} \in \mathbb{C}\left[x_{1}, x_{2}, x_{3}, x_{4}\right]$, so $x+H$ is invertible. Since $H$ is homogeneous of degree 6 , the nilpotency of $\mathcal{J} H$ follows.

To show the linear independence of the components of $H$, put $y=\left(t+1, t^{2}, t^{3}, t^{4}\right.$, 1). Then $a(y)=t^{4}$ and the leading terms to $t$ in the components of $H(y)$ are

$$
t^{5}, t^{9}, t^{7}, t^{11}, t^{12}
$$

whence the components of $H$ are linearly independent.

Corollary 3.3. Let $n \geq 6$ and put

$$
H=\left(x_{5} H_{x_{5}, x_{6}}^{[4]}, a^{2}, x_{5}^{4}, \ldots, x_{n-1}^{4}\right) .
$$

Then $H$ is a counterexample of degree 4 to the Linear Dependence Problem for Homogeneous Jacobians.

Proof. Again we apply Theorem 3.1 with $s=4$. The nilpotency of $\mathcal{J} H$ follows in a similar matter as in the previous corollary.

To show the linear independence of the components of $H$, put $y=\left(t, t^{2}, t^{3}, t^{4}+1\right.$, $\left.1, t^{6}, t^{7}, \ldots, t^{n}\right)$. Then $a(y)=t$, and the leading terms to $t$ of $H(y)$ are

$$
-t^{14},-t^{8},-t^{16},-t^{10}, t^{8}, 1, t^{24}, \ldots, t^{4(n-1)},
$$

whence the components of $H$ are linearly indepenent.

Corollary 3.4. Let $n \geq 10$ and put

$$
H=\left(H_{x_{9}, x_{10}}^{[6]}, x_{9} a, x_{9} b, x_{8} a-x_{7} b, x_{9}^{3}, x_{10}^{3}, \ldots, x_{n-1}^{3}\right) .
$$

Then $H$ is a cubic counterexample to the Linear Dependence Problem for Homogeneous Jacobians.

Proof. We apply Theorem 3.1 with $s=8$, so let

$$
D=\sum_{i=1}^{8} H_{i} \frac{\partial}{\partial x_{i}}
$$

From $a, b \in \operatorname{ker} D$ and the construction of $H_{7}$ and $H_{8}, H_{9} \in \operatorname{ker} D$ follows. So the conditions of Theorem 3.1 are fulfilled and $\mathcal{J} H$ is nilpotent. 
Put $y=\left(t, t^{2}, t^{3}+1, t^{4}, t^{5}, t^{6}, t^{7}, t^{8}, 1, t^{10}, t^{11}, \ldots, t^{n}\right)$. Then $a(y)=-t^{2}$ and $b(y)=t^{6}$. So the leading terms to $t$ of $H(y)$ are

$$
-t^{22},-t^{12},-t^{24},-t^{14},-t^{26},-t^{16},-t^{2}, t^{6},-t^{13}, 1, t^{30}, \ldots, t^{3(n-1)} .
$$

Therefore, the components of $H$ are linearly independent.

\section{FinAL REMARKS}

In [2, techniques are given to make Jacobians symmetric by way of stabilization, such that the rows of the Jacobian remain linearly independent over $\mathbb{C}$. The Jacobian becomes a Hessian, i.e. the Jacobian of a gradient.

Assume we have a nilpotent Jacobian $\mathcal{J} H$ with $H=\left(H_{1}, H_{2}, \ldots, H_{n}\right)$, whose rows are linearly independent over $\mathbb{C}$. Then the columns do not need to be linearly independent. But if $\mathcal{J} H \cdot \lambda=0$ for some nonzero $\lambda \in \mathbb{C}^{n}$, then each $H_{i}$ is contained in the kernel of

$$
D=\sum_{i=1}^{n} \lambda_{i} \frac{\partial}{\partial x_{i}}
$$

Now there is a linear coordinate system $y=y_{1}, y_{2}, \ldots, y_{n}$ such that $y_{i} \in \operatorname{ker} D$ for all $i \leq n-1$, whence $H$ can be expressed as a polynomial map in $y_{1}, y_{2}, \ldots, y_{n-1}$. So the last column of $\mathcal{J}_{y} H$ is zero, where $\mathcal{J}_{y}$ is the Jacobian with respect to $y$. Proceeding this way, we can ensure that the first $s$ columns of $\mathcal{J}_{z}(H)$ are linearly independent over $\mathbb{C}$ and the last $n-s$ columns of $\mathcal{J}_{z}(H)$ are zero for some linear coordinates system $z$, i.e. $H_{i} \in \mathbb{C}\left[z_{1}, z_{2}, \ldots, z_{s}\right]$ for all $i$.

Theorem 4.1. Assume $H \in \mathbb{C}\left[x_{1}, x_{2}, \ldots, x_{s}\right]^{n}$ has a nilpotent Jacobian. Assume further that both the rows of $\mathcal{J} H$ and the first $s$ columns of $\mathcal{J} H$ are linearly independent over $\mathbb{C}$. Then

$$
h=\sum_{i=1}^{n} x_{i} H_{i}\left(x_{1}+\mathrm{i} x_{n+1}, x_{2}+\mathrm{i} x_{n+2}, \ldots, x_{s}+\mathrm{i} x_{n+s}\right)+\sum_{i=s+1}^{m}\left(x_{i}+\mathrm{i} x_{n+i}\right)^{d+1}
$$

has a nilpotent Hessian if $s \leq m \leq n$. Furthermore, the rows of $\mathcal{H} h=\mathcal{J}(\nabla h)$ are linearly independent. Moreover, $h$ is homogeneous in case $H$ is homogeneous of degree $d$.

This theorem can be proved with techniques presented in [2].

Talking about Hessians, you might wonder why P. Gordan and and M. Nöther were interested in (homogeneous) quasi-translations. The reason is that they were studying (homogeneous) Hessians with determinant zero. For such Hessians $\mathcal{H} h$, there exists a (homogeneous) relation $R$ between the components of $\nabla h$. Now if $h \in \mathbb{C}[x]=\mathbb{C}\left[x_{1}, x_{2}, \ldots, x_{n}\right]$, then $x+H$ with

$$
H=\nabla R \circ \nabla h
$$

is a quasi-translation; see [3] or just the original paper 9]. It is remarkable that P. Gordan and M. Nöther already juggled with nilpotent derivations before derivations were invented.

For quadratic homogeneous maps, in particular quadratic linear maps, the linear dependence problem was first stated as Conjecture 11.3 by K. Rusek in [13]. This conjecture is still open. 


\section{ACKNOWLEDGMENT}

The author thanks A. van den Essen for his idea of debunking the Homogeneous Dependence Problem by examining quasi-translations.

\section{REFERENCES}

[1] H. Bass, E. Connel and D. Wright, The Jacobian Conjecture: Reduction of Degree and Formal Expansion of the Inverse, Bull. of the AMS, 7 (1982), 287-330. MR0663785 (83k:14028)

[2] M. de Bondt and A. van den Essen, A reduction of the Jacobian Conjecture to the symmetric case, Proc. Amer. Math. Soc., 133 (2005), 2201-2205. MR2138860

[3] M. de Bondt and A. van den Essen, Singular Hessians, J. Algebra, 282 (2004), 195-204. MR2095579 (2005i:13031)

[4] M. de Bondt and A. van den Essen, The Jacobian Conjecture: linear triangularization for homogeneous polynomial maps in dimension three, J. Algebra, 294 (2005), 294-306. MR2179727

[5] M. de Bondt, Homogeneous quasi-translations and an article of P. Gordan and M. Nöther, Report 0417, Dep. Math., Radboud University Nijmegen.

[6] A. Cima, A. Gasull and F. Mañosas, The discrete Markus-Yamabe problem, Nonlinear Analysis: Theory, Methods \& Applications, 35 (1999), no. 3, pp. 343-354. MR 1643454(2000j:37030)

[7] A. van den Essen, Polynomial Automorphisms and the Jacobian Conjecture, Vol. 190 in Progress in Mathematics, Birkhäuser, 2000. MR1790619(2001j:14082)

[8] G. Freudenburg, Actions of $G_{a}$ on $\mathbb{A}^{3}$ defined by homogeneous derivations, J. of Pure and Applied Algebra, 126 (1998), 169-181. MR.1600530 (98k:14068)

[9] P. Gordan and M. Nöther, Über die algebraische Formen, deren Hesse'sche Determinante identisch verschwindet, Mathematische Annalen, 10 (1876), 547-568. MR.1509898

[10] E. Hubbers, The Jacobian Conjecture: Cubic homogeneous maps in Dimension Four, Master's thesis, Univ. of Nijmegen, 1994.

[11] G.H. Meisters, Polyomorphisms conjugate to Dilations, In Automorphisms of Affine Spaces, Curaçao, July 4-8, 1994, Caribean Mathematics Foundation, Kluwer Academic Press, 1995, Proceedings of the conference 'Invertible Polynomial maps'. MR 1352691 (97m:14020)

[12] C. Olech, On Markus-Yamabe stability conjecture, Proc. of the Intern. Meeting on Ordinary Differential Equations and their Applications, Univ. of Florence, 1995, pp. 127-137.

[13] K. Rusek, Polynomial Automorphisms, preprint 456, Institute of Mathematics, Polish Academy of Sciences, IMPAN, Śniadeckich 8, P.O. Box 137, 00-950 Warsaw, Poland, May 1989.

[14] Z. Wang, Homogeneization of Locally Nilpotent Derivations and an Application to $k[X, Y, Z]$, J. Pure Appl. Algebra, 196 (2005), 323-337. MR2110528 (2005j:13025)

Department of Mathematics, Radboud University, Postbus 9010, 6500 GL Nijmegen, The Netherlands

E-mail address: debondt@math.ru.nl 